\title{
versants
}

\section{Letteratura e dialetti. Gli ultimi decenni: Premessa}

\author{
Stefano BARELLI \\ Université de Fribourg
}

\begin{abstract}
Versants si propone di esaminare lo statuto e le funzioni del dialetto nella letteratura italiana contemporanea. A essere considerata dagli studiosi non è solo la produzione dialettale in senso stretto ma anche l'ibridazione che, tanto nella poesia quanto nella prosa, costituisce un tratto specifico e per molti versi inedito della nostra cultura linguistica e letteraria.
\end{abstract}

Keywords: relazione lingua-dialetto, mescolanze ed ibridazioni, multilinguismo, nuovo statuto del dialetto.

Nel 1999, a introduzione dei tre volumi che costituiscono la prima sistematica rassegna delle letterature in dialetto delle regioni italiane, Franco Brevini constatava come i poeti dialettali contemporanei scrivano ormai in absentia: praticamente scomparsa la cultura popolare che lo aveva generato e di cui era lo strumento espressivo, il dialetto non può essere assunto che come «lingua della memoria» (o anche, secondo Pier Vincenzo Mengaldo, come «ritorno del represso», inteso in senso sia psicologico che sociale: Brevini 1999: I, XCVII; Mengaldo 2000: 8).

A distanza di quasi vent'anni da queste constatazioni, e alla luce dei più recenti sviluppi della lingua e della società, è opportuno tornare a interrogarsi sullo statuto e la funzione del dialetto nella letteratura: includendo nella valutazione anche la dimensione della prosa, forse in passato troppo trascurata e dove la naturale mescidazione con la lingua sottrae il vernacolo a quella sublimazione che costituisce forse il comun denominatore di gran parte dell'attuale poesia in dialetto (con il costante rischio della fuga verso l'idillio, come denunciato ad esempio da Fernando Bandini: si veda qui il contributo di Rodolfo Zucco). La tendenza alla conservazione di parlate locali a integrazione della lingua e la conseguente (e forse crescente) assunzione di componenti dialettali nell'italiano standard osservata dai linguisti (De Mauro 20I4: III-I32) ha in effetti prodotto, dalla fine dello scorso millennio, effetti di grande portata non solo nella produzione letteraria ma anche nella sua ricezione e diffusione presso il pubblico dei lettori (il caso Camilleri è in questo senso emblematico).

Il presente fascicolo di Versants intende sondare queste nuove tendenze, prendendo in considerazione tanto la produzione dialettale in senso stretto quanto le diverse manifestazioni di un'ibridazione linguistica che costituisce uno dei tratti più rilevanti della cultura italiana contemporanea. Riguar- 
do a quest'ultimo punto Edoardo Zuccato rileva, analizzando la produzione di alcuni giovani poeti, come la globalizzazione in atto stia intaccando profondamente il classico dualismo italiano-dialetto, finora cardine essenziale della nostra storia linguistica e letteraria: al suo posto va delineandosi un multilinguismo la cui fisionomia non è ancora precisamente tracciabile e che potrebbe decretare la conclusione della cosiddetta questione della lingua.

Il rapporto tra lingua e dialetto in due poeti «bilingui» come Giorgio Orelli e Fernando Bandini è sondato nei contributi di Ariele Morinini e di Rodolfo Zucco: il primo indaga il ruolo e il rilievo (progressivamente crescente) del dialetto all'interno di una produzione prevalentemente in lingua; il secondo si interroga sulle funzioni e le ragioni del vernacolo nell'opera poetica di un letterato che, paradossalmente, diffidava e metteva in guardia circa l'impiego del dialetto in poesia. Jean-Jacques Marchand analizza l'effetto straniante e la funzione «civile» prodotti dall'ampio impiego di elvetismi e di toponomia locale nella poesia italiana di Alberto Nessi. La costituzione di piccoli «canzonieri» dialettali da parte di Franco Loi e di Leo Zanier è trattata nei saggi di Pietro Montorfani e Ottavio Besomi, che esaminano le strutture delle raccolte e il rapporto con la tradizione. Clelia Martignoni indica alcuni percorsi critici ancora inesplorati a proposito della poesia di uno dei grandi esponenti della letteratura dialettale moderna, Raffaello Baldini.

Il versante della prosa è indagato da Georgia Fioroni, Luciano Zampese e Diego Varini, che analizzano l'incontro-scontro e il pastiche tra lingua standard e idioma regionale che caratterizza tanta parte della narrativa contemporanea: sotto la lente degli studiosi sono posti il tema dell'emigrazione, soprattutto nella narrativa di Antonio Pennacchi, intesa come trasferimento anche linguistico con l'ibridazione che ne consegue (Fioroni); e l'impasto di dialetto e lingua nell'opera di Luigi Meneghello, di cui vengono presentati anche materiali inediti (Zampese), e di Michele Mari (Varini). Chiude il fascicolo, nella sezione «Contributi vari», il saggio di Matteo M. Pedroni sulla funzione dei ricordi d'infanzia nell'opera letteraria e saggistica di Giosuè Carducci.

Spiace che non sia stato possibile includere studi specifici relativi all'impiego letterario del dialetto nell'area meridionale, che avrebbero permesso un più esaustivo confronto anche sul piano geografico tra le tendenze attualmente in corso: ma, lungi dall'ambire ad un'impossibile esaustività, questo numero di Versants intende piuttosto proporre una prima e provvisoria mappa, tracciata mediante campionature e indagini mirate, di territori che altri provvederà a contornare, arricchire e sistematizzare. 


\section{Bibliografia}

Brevini, Franco, La poesia in dialetto. Storia e testi dalle origini al Novecento, 3 vol., Milano, Mondadori, 1999.

De Mauro, Tullio, Storia linguistica dell'Italia repubblicana dal 1946 ai giorni nostri, Roma-Bari, Laterza, 20I4.

Mengaldo, Pier Vincenzo, «Problemi della poesia dialettale italiana del Novecento», La tradizione italiana del Novecento. Quarta serie, Torino, Bollati Boringhieri, 2000, pp. 3-I4. 